\title{
Misconceptions on the application of biological market theory to the mycorrhizal symbiosis
}

E. Toby Kiers ${ }^{1}$, Stuart A. West ${ }^{2}$, Gregory A.K. Wyatt ${ }^{2}$, Andy Gardner ${ }^{3}$ Heike Bücking ${ }^{4}$, and Gijsbert D.A. Werner ${ }^{1}$

1Department of Ecological Sciences, Vrije Universiteit, De Boelelaan 1085, 1081 HV Amsterdam, The Netherlands

${ }^{2}$ Department of Zoology, University of Oxford, South Parks Road, Oxford OX1 3PS, United Kingdom.

${ }^{3}$ School of Biology, University of St Andrews, Dyers Brae, St Andrews KY16 9TH United Kingdom 4Biology and Microbiology Department, South Dakota State University, Brookings, SD 57007, USA

\section{Letter to Editor}

The symbiosis between plants and arbuscular mycorrhizal fungi has been described as a biological market based on evidence that plants supply more carbohydrates to fungal partners that provide more soil nutrients, and vice versa1-4. Walder \& Van der Heijden's (WH) recent paper challenges this view ${ }^{5}$. However, WH's challenge is based upon misunderstandings of biological market theory, and evolutionary theory more generally.

First, WH's claim that biological market theory requires (or assumes) tightly-coupled direct resource exchange is incorrect. All that is required is that individuals have a preference on average for interacting with more beneficial partners ${ }^{6-9}$. Biological market theory makes no claim on understanding (proximate) mechanisms of transfer processes. This is not its aim. Instead, biological market theory addresses ultimate questions such as why partnerships remain stable over evolutionary time, even in the presence of less beneficial partners. Its usefulness lies in predicting how these exchanges will be affected by context, such as varying environmental conditions ${ }^{7,8,10-12}$.

Second, WH suggest that the "most prominent fact" challenging a market-based view of the mutualism is the occurrence of antagonistic or parasitic interactions. However, this claim reveals a key misunderstanding of biological market theory, and mutualism evolution in general. The persistence of some parasitism is actually expected under biological market theory, which predicts neither complete disappearance of cheating nor perfect partner choice, $8,9,13,14$. In line with this prediction, we observe that parasitic interactions remain a small, but observable, proportion of global mycorrhizal interactions ${ }^{15}$.

Third, the authors claim that plant-derived carbon is available to fungal symbionts as a "public good" (i.e. individuals cannot be effectively excluded from its use). Both theory and physiology argue against this scenario. Theory unambiguously predicts that symbiotic persistence is facilitated by the evolution of adaptations to exclude less beneficial partners, such that free access to resources is restricted ${ }^{16-18}$. This is backed by physiological evidence of (1) localized carbon delivery to the fungus, namely the evolution of intracellular structures (e.g. arbuscules and coils) that facilitate the directed transfer of nutrients, and (2) knockdown and genesilencing studies suggesting cell-specific nutrient supply determines arbuscule longevity (19 for review). 
Fourth, WH claim that the lack of specificity in the mycorrhizal symbiosis is evidence against the existence of partner choice. This claim is based on a misunderstanding of the existing theory, which predicts the opposite. Biological market theory predicts that low specificity, with a higher number of potential partners, facilitates the evolution of stable trading $6,7,20$. As a host you can gain more when you have the potential to interact with multiple fungal strains, in contrast to being 'locked-in' to one partner ${ }^{7,20}$. This is especially true in variable environmental conditions ${ }^{10,11}$.

Fifth, the authors argue that in complex networks (i.e. multiple plants and multiple fungi), partner choice may be "difficult and less effective". This claim is likewise based on a misunderstanding of existing theory, which makes the opposite prediction? ${ }^{7}$ The authors also argue that "spatial separation may serve as a critical precondition" for discrimination 5 . However, there are mixed findings on the importance of spatial structure, both experimentally $1,2,21$ and theoretically ${ }^{8,21,22}$, but the variation we find in the precision of partner choice among species ${ }^{22}$ and under different conditions ${ }^{4,24}$ is actually expected, and again consistent with theory ${ }^{9}$.

Sixth, WH suggest it is a problem that partner identity, environmental conditions, and available external resources all vary in ways that could affect trading choices. But this is not a problem this is how markets work, both in theory and in practice $6-8,10,11,24$. In contrast to what the authors claim, variable rewards and changing partner preferences are the defining feature of biological markets $6,7,19$.

The goal of biological market theory is not to draw analogies to human markets. Rather, biological market theory is a tool to analyse exchange patterns. When applied correctly, it allows scientists to make testable predictions about resource exchange patterns, and how these vary across species and environment ${ }^{10,12,20,25}$. In the past, mycorrhizal researchers could only vaguely refer to "context-dependency" to explain the variability in their results. Biological market theory now allows us to dissect this variability and generate specific and precise predictions for plant-mycorrhizal outcomes $7,8,11,12,20$.

References:

1. Bever, J. D., Richardson, S. C., Lawrence, B. M., Holmes, J. \& Watson, M. Preferential allocation to beneficial symbiont with spatial structure maintains mycorrhizal mutualism. Ecol. Lett. 12, 13-21 (2009).

2. Kiers, E. T. et al. Reciprocal Rewards Stabilize Cooperation in the Mycorrhizal Symbiosis. Science 333, 880-882 (2011).

3. Fellbaum, C. R. et al. Carbon availability triggers fungal nitrogen uptake and transport in arbuscular mycorrhizal symbiosis. Proc. Natl. Acad. Sci. 109, 2666-2671 (2012).

4. Fellbaum, C. R. et al. Fungal nutrient allocation in common mycorrhizal networks is regulated by the carbon source strength of individual host plants. New Phytol. 203, 646656 (2014).

5. Walder, F. \& van der Heijden, M. G. A. Regulation of resource exchange in the arbuscular mycorrhizal symbiosis. Nat. Plants 1, 15159 (2015).

6. Noë, R. \& Hammerstein, P. Biological markets: supply and demand determine the effect of partner choice in cooperation, mutualism and mating. Behav. Ecol. Sociobiol. 35, 1-11 (1994).

7. Wyatt, G. A. K., Kiers, E. T., Gardner, A. \& West, S. A. A biological market analysis of the plant-mycorrhizal symbiosis. Evol. 68, 2603-2618 (2014). 
8. Bever, J. D. Preferential allocation, physio-evolutionary feedbacks, and the stability and environmental patterns of mutualism between plants and their root symbionts. New Phytol. 205, 1503-1514 (2015).

9. Steidinger, B. S. \& Bever, J. D. The Coexistence of Hosts with Different Abilities to Discriminate against Cheater Partners: An Evolutionary Game-Theory Approach. Am. Nat. 183, 762-70 (2014).

10. Werner, G. D. A. et al. Evolution of microbial markets. Proc. Natl. Acad. Sci. 111, 12371244 (2014).

11. Werner, G. D. A. \& Kiers, E. T. Partner selection in the mycorrhizal mutualism. New Phytol. 205, 1437-1442 (2015).

12. Johnson, N. C. Resource stoichiometry elucidates the structure and function of arbuscular mycorrhizas across scales. New Phytol. 185, 631-47 (2010).

13. Song, Z. \& Feldman, M. W. Plant-animal mutualism in biological markets: Evolutionary and ecological dynamics driven by non-heritable phenotypic variance. Theor. Popul. Biol. 88, 20-30 (2013).

14. Foster, K. R. \& Kokko, H. Cheating can stabilize cooperation in mutualisms. Proc. R. Soc. B Biol. Sci. 273, 2233-2239 (2006).

15. Hoeksema, J. D. et al. A meta-analysis of context-dependency in plant response to inoculation with mycorrhizal fungi. Ecol. Lett. 13, 394-407 (2010).

16. Frank, S. a. A general model of the public goods dilemma. J. Evol. Biol. 23, 1245-1250 (2010).

17. West, S. A., Griffin, A. S. \& Gardner, A. Evolutionary Explanations for Cooperation. Curr. Biol. 17, R661-R672 (2007).

18. Ghoul, M., Griffin, A. S. \& West, S. A. Toward an evolutionary definition of cheating. Evolution 68, 318-331 (2014).

19. Gutjahr C. \& Parniske M. Cell and developmental biology of arbuscular mycorrhiza symbiosis. Ann. Rev. Cell Dev. Biol. 29, 593-617 (2013).

20. Kummel, M. \& Salant, S. W. The economics of mutualisms: optimal utilization of mycorrhizal mutualistic partners by plants. Ecology 87, 892-902 (2006).

21. Verbruggen, E. et al. Spatial Structure and Interspecific Cooperation: Theory and an Empirical Test Using the Mycorrhizal Mutualism. Am. Nat. 179, E133-E146 (2012).

22. Steidinger, B. S. \& Bever, J. D. Host discrimination in modular mutualisms: a theoretical framework for meta-populations of mutualists and exploiters. Proc. R. Soc. B Biol. Sci. 283, 20152428 (2016).

23. Grman, E. Plant species differ in their ability to reduce allocation to non-beneficial arbuscular mycorrhizal fungi. Ecology 93, 711-718 (2012).

24. Zheng, C., Ji, B., Zhang, J., Zhang, F. \& Bever, J. D. Shading decreases plant carbon preferential allocation towards the most beneficial mycorrhizal mutualist. New Phytol. 205, 361-368 (2015).

25. Schwartz, M. W. \& Hoeksema, J. D. Specialization and resource trade: Biological markets as a model of mutualisms. Ecology 79, 1029-1038 (1998). 